\title{
Geometric Foundations of Numerical Algorithms and Symmetry
}

\author{
Peter J. Olver ${ }^{\dagger}$ \\ School of Mathematics \\ University of Minnesota \\ Minneapolis, MN 55455 \\ U.S.A. \\ olver@umn.edu \\ http: //www . math. umn. edu/ olver
}

\begin{abstract}
This paper outlines a new general construction, named "multi-space", that forms the proper geometrical foundation for the numerical analysis of differential equations - in direct analogy with the role played by jet space as the basic object underlying the geometry of differential equations. Application of the theory of moving frames leads to a general framework for constructing symmetry-preserving numerical approximations to differential invariants and invariant differential equations.
\end{abstract}

$\dagger$ Supported in part by NSF Grant DMS 11-08894.

December 20, 2020 


\section{Introduction.}

Despite being in extensive, albeit implicit, use since the days of Lie and Cartan, jet space was first formally defined by Ehresmann, [13], to serve as the proper foundation for studying the geometry of differential equations. The purpose of this paper is to establish a new construction, called "multi-space", that plays the same geometric, foundational role for the numerical analysis of differential equations. The simplest example of a multi-space already appears in the classical blow-up construction in algebraic geometry, [16], that forms the basis of Hironaka's resolution of singularities, [18]. Multi-space is a far reaching generalization of this classical construction, and has interesting, but as yet unexplored, connections with the Hilbert scheme of points on an algebraic variety, [19]. In particular, the multi-space formulation permits the direct application of the new method of moving frames, developed in collaboration with Mark Fels, $[\mathbf{1 4}, \mathbf{1 5}]$, to the systematic construction of invariant numerical approximations to differential invariants and invariant differential equations.

In modern numerical analysis, the development of numerical schemes that incorporate additional structure enjoyed by the problem being approximated have become quite popular in recent years. The first instances of such schemes are the symplectic integrators arising in Hamiltonian mechanics, and the related energy conserving methods, $[\mathbf{8 , 2 3}, \mathbf{3 2}]$. The design of symmetry-based numerical approximation schemes for differential equations has been studied by various authors, including Shokin, $[\mathbf{3 1}]$, Dorodnitsyn, $[\mathbf{1 1}, \mathbf{1 2}]$, Axford and Jaegers, $[\mathbf{2 1}]$, and Budd and Collins, $[\mathbf{3}]$. These methods are closely related to the active area of geometric integration of ordinary differential equations on Lie groups, $[\mathbf{4}, \mathbf{2 0}, \mathbf{2 4}]$. In practical applications of invariant theory to computer vision, group-invariant numerical schemes to approximate differential invariants based on suitable combinations of joint invariants, [29], have been applied to the problem of symmetry-based object recognition, $[2,5,6]$.

This paper serves as a brief introduction to the basic ideas of multi-space and invariant numerical algorithms. Owing to significant multi-dimensional complications, only the simplest case of curves - by which we always mean embedded one-dimensional submanifolds - and ordinary differential equations will be treated here. Moreover, the practical evaluation of these numerical schemes will also be deferred until a more extensive investigation is completed. Thus, this note is not meant to be a definitive treatment of either subject, but rather a report of work in progress.

\section{Multi-Space for Curves.}

In this section, we outline the basic construction of multi-space that forms the foundation for the study of the geometric properties of discrete approximations to derivatives and numerical solutions to differential equations. We will only discuss the case of curves, which correspond to functions of a single independent variable, and hence satisfy ordinary differential equations. The more difficult case of higher dimensional submanifolds, corresponding to functions of several variables that satisfy partial differential equations, relies

on a new approach to multi-dimensional interpolation theory, $[\mathbf{3 0}]$, and its construction remains an open problem. We will state results in the smooth, meaning $\mathrm{C}^{\infty}$, category; all 
results have immediate analytic counterparts, and one can, with proper care, also formulate analogous constructions for curves with only finite order of differentiability.

Given a manifold $M$, we let $\mathrm{J}^{n}=\mathrm{J}^{n}(M, 1)$ denote the extended ${ }^{\dagger} n^{\text {th }}$ order jet space for one-dimensional submanifolds $C \subset M$. Jet space is defined as the space of equivalence classes of curves under the equivalence relation of $n^{\text {th }}$ order contact at a single point. We let $\left.\mathrm{j}_{n} C\right|_{z}$ denote the $n$-jet or equivalence class of the curve $C$ at the point $z \in C$. If we introduce local coordinates $z=(x, u)=\left(x, u^{1}, \ldots, u^{q}\right)$, where $q=\operatorname{dim} M-1$, on a coordinate chart $\widetilde{M} \subset M$ then a curve $C=\{u=f(x)\}$ defined by a smooth function $f$ will be called a graph. The graphs are transverse to the vertical fibers $\{x=c\}$, and so their jets define an open dense coordinate chart for the restriction of $\mathrm{J}^{n}$ to $\widetilde{M}$. The corresponding jet coordinates of $\mathrm{j}_{n} C$ are the derivatives $u^{(n)}=f^{(n)}(x)$ for $n=0,1,2, \ldots$

Numerical finite difference approximations to the derivatives of a function $u=f(x)$ rely on its values $u_{0}=f\left(x_{0}\right), \ldots, u_{n}=f\left(x_{n}\right)$ at several distinct points $z_{i}=\left(x_{i}, u_{i}\right)=$ $\left(x_{i}, f\left(x_{i}\right)\right)$ on the curve. Thus, discrete approximations to jet coordinates on $\mathrm{J}^{n}$ are functions $F\left(z_{0}, \ldots, z_{n}\right)$ defined on the $(n+1)$-fold Cartesian product space $M^{\times(n+1)}=$ $M \times \cdots \times M$. Note that to adequately approximate a derivative of order $n$ requires at least $n+1$ function values, so the numerology is correct.

In order to seamlessly connect the jet coordinates with their discrete approximations, then, we need to relate the jet space $\mathrm{J}^{n}$ to the Cartesian product space $M^{\times(n+1)}$. Now, as the points $z_{0}, \ldots, z_{n}$ coalesce, the approximation $F\left(z_{0}, \ldots, z_{n}\right)$ will not be well-defined unless we specify the "direction" of convergence. Thus, strictly speaking, $F$ is not defined on all of $M^{\times(n+1)}$, but, rather, on the "off-diagonal" part, by which we mean the subset

$$
M^{\diamond(n+1)}=\left\{\left(z_{0}, \ldots, z_{n}\right) \mid z_{i} \neq z_{j} \text { for all } i \neq j\right\} \subset M^{\times(n+1)}
$$

consisting of all distinct $(n+1)$-tuples of points. As two or more points come together, the limiting value of $F\left(z_{0}, \ldots, z_{n}\right)$ will be governed by the derivatives (or jet) of the appropriate order governing the direction of convergence. This observation serves to motivate our construction of the $n^{\text {th }}$ order multi-space $M^{(n)}$, which shall contain both the jet space $\mathrm{J}^{n}$ and the off-diagonal Cartesian product space $M^{\diamond(n+1)}$ in a consistent manner.

Definition 2.1. An $(n+1)$-pointed manifold is an object $\mathbf{M}=\left(z_{0}, \ldots, z_{n} ; M\right)$ consisting of a smooth manifold $M$ and $n+1$ not necessarily distinct points $z_{0}, \ldots, z_{n} \in M$ thereon. Given $\mathbf{M}$, we let $\# i=\#\left\{j \mid z_{j}=z_{i}\right\}$ denote the number of points which coincide with the $i^{\text {th }}$ one.

Given a manifold $M$, we let $\mathcal{C}^{(n)}=\mathcal{C}^{(n)}(M)$ denote the set of all $(n+1)$-pointed curves contained in $M$. We define an equivalence relation on the space of multi-pointed curves that generalizes the jet equivalence relation of $n^{\text {th }}$ order contact at a single point.

Definition 2.2. Two $(n+1)$-pointed curves

$$
\mathbf{C}=\left(z_{0}, \ldots, z_{n} ; C\right), \quad \widetilde{\mathbf{C}}=\left(\tilde{z}_{0}, \ldots, \tilde{z}_{n} ; \widetilde{C}\right)
$$

$\dagger$ The adjective "extended" refers to the fact that we are allowing arbitrary curves and not just those satisfying some sort of transversality condition. See [27; Chapter 3] for details. 
have $n^{\text {th }}$ order multi-contact if and only if

$$
z_{i}=\tilde{z}_{i}, \quad \text { and }\left.\quad \mathrm{j}_{\# i-1} C\right|_{z_{i}}=\left.\mathrm{j}_{\# i-1} \widetilde{C}\right|_{z_{i}}, \quad \text { for each } \quad i=0, \ldots, n .
$$

The $n^{\text {th }}$ order multi-space, denoted $M^{(n)}$ is the set of equivalence classes of $(n+1)$-pointed curves in $M$ under the equivalence relation of $n^{\text {th }}$ order multi-contact. The equivalence class of an $(n+1)$-pointed curves $\mathbf{C}$ is called its $n^{\text {th }}$ order multi-jet, and denoted $\mathbf{j}_{n} \mathbf{C} \in M^{(n)}$.

In particular, if the points on $\mathbf{C}=\left(z_{0}, \ldots, z_{n} ; C\right)$ are all distinct, then $\mathbf{j}_{n} \mathbf{C}=\mathbf{j}_{n} \widetilde{\mathbf{C}}$ if and only if $z_{i}=\tilde{z}_{i}$ for all $i$, which means that $\mathbf{C}$ and $\widetilde{\mathbf{C}}$ have all $n+1$ points in common. Therefore, we can identify the subset of multi-jets of multi-pointed curves having distinct points with the off-diagonal Cartesian product space $M^{\diamond(n+1)} \subset \mathrm{J}^{n}$. On the other hand, if all $n+1$ points coincide, $z_{0}=\ldots=z_{n}$, then $\mathbf{j}_{n} \mathbf{C}=\mathbf{j}_{n} \widetilde{\mathbf{C}}$ if and only if $\mathbf{C}$ and $\widetilde{\mathbf{C}}$ have $n^{\text {th }}$ order contact at their common point $z_{0}=\tilde{z}_{0}$. Therefore, the multi-space equivalence relation reduces to the ordinary jet space equivalence relation on the set of coincident multi-pointed curves, and in this way $\mathrm{J}^{n} \subset M^{(n)}$. These two extremes do not exhaust the possibilities, since one can have some but not all points coincide. Intermediate cases correspond to "off-diagonal" Cartesian products of jet spaces

$$
\mathrm{J}^{k_{1}} \diamond \cdots \diamond \mathrm{J}^{k_{i}} \equiv\left\{\left(z_{0}^{\left(k_{1}\right)}, \ldots, z_{i}^{\left(k_{i}\right)}\right) \in \mathrm{J}^{k_{1}} \times \cdots \times \mathrm{J}^{k_{i}} \mid \pi\left(z_{\nu}^{\left(k_{\nu}\right)}\right) \text { are distinct }\right\},
$$

where $\sum k_{\nu}=n$ and $\pi: \mathrm{J}^{k} \rightarrow M$ is the usual jet space projection. These multi-jet spaces appear in the work of Dhooghe, [10], on the theory of "semi-differential invariants" in computer vision. For instance, we can decompose

$$
M^{(3)}=M^{\diamond 4} \cup 6\left(M \diamond M \diamond \mathrm{J}^{1}\right) \cup 3\left(\mathrm{~J}^{1} \diamond \mathrm{J}^{1}\right) \cup 4\left(M \diamond \mathrm{J}^{2}\right) \cup \mathrm{J}^{3}
$$

into a disjoint union, where, for instance, the factor

$$
\left.\mathrm{J}^{1} \diamond \mathrm{J}^{1}=\left\{\left(z_{0}^{(1)}, z_{1}^{(1)}\right) \in \mathrm{J}^{1} \times \mathrm{J}^{1}\right) \mid \pi\left(z_{0}^{(1)}\right) \neq \pi\left(z_{1}^{(1)}\right)\right\}
$$

corresponds to 4 -pointed curves $\left(z_{0}, z_{1}, z_{2}, z_{3} ; C\right)$ with $z_{0}=z_{1} \neq z_{2}=z_{3}$, and appears 3 distinct times, depending on which pairs of points coincide.

An outline of the proof of the following fundamental result appears in the following section.

Theorem 2.3. If $M$ is a smooth $m$-dimensional manifold, then its $n^{\text {th }}$ order multispace $M^{(n)}$ is a smooth manifold of dimension $(n+1) m$, which contains the off-diagonal part $M^{\diamond(n+1)}$ of the Cartesian product space as an open, dense submanifold, and the $n^{\text {th }}$ order jet space $\mathrm{J}^{n}$ as a smooth submanifold.

Remark: The action of the permutation group $\mathbb{S}^{n+1}$ on the points of our multi-pointed curves induces an action on $M^{(n)}$. Many (but by no means all) objects of interest are invariant under this permutation group action. Unfortunately, the quotient space $M^{(n)} / \mathbb{S}^{n+1}$ is, in general, only an "orbifold" with boundary. 
Example 2.4. If $M=\mathbb{R}^{m}$ is a Euclidean space, then the first order multi-space $M^{(1)}$ is constructed from the class of 2-pointed curves $\mathcal{C}^{(1)}$ by using the equivalence relation $\left(z_{0}, z_{1} ; C\right) \sim\left(\tilde{z}_{0}, \tilde{z}_{1}, \widetilde{C}\right)$ if and only if either

(a) $z_{0}=\tilde{z}_{0} \neq z_{1}=\tilde{z}_{1}$, and so $C$ and $\widetilde{C}$ have zero ${ }^{\text {th }}$ order contact at their two common points, or

(b) $z_{0}=\tilde{z}_{0}=z_{1}=\tilde{z}_{1}$ and $C$ and $\widetilde{C}$ have first order contact at this common point. We can thus identify $M^{(1)}$ with the space of two-pointed lines, i.e.,

$$
M^{(1)} \simeq\left\{\left(z_{0}, z_{1} ; L\right) \mid z_{0}, z_{1} \in L, \text { where } L \subset \mathbb{R}^{m} \text { is a line }\right\} .
$$

If $z_{0} \neq z_{1}$, then $L$ is uniquely determined; otherwise we use $L$ to prescribe the tangent direction at the point $z_{0}=z_{1}$. The reader may compare this with the method of "blowing up" singularities in algebraic geometry, [16], which, indeed, can be viewed as a very particular case of our general multi-space construction. Similarly, we can identify $M^{(2)}$ with the space of three-pointed circles, i.e.,

$$
M^{(2)} \simeq\left\{\left(z_{0}, z_{1}, z_{2}, C\right) \mid z_{0}, z_{1}, z_{2} \in C, \text { where } C \subset \mathbb{R}^{m} \text { is a circle }\right\} .
$$

Straight lines are included as circles of infinite radius, but points are not included (even though they could be viewed as circles of zero radius). This relies on the fact that a circle in Euclidean space is uniquely determined by three non-coincident points, or by a point and a tangent direction at a second point, or by a single osculating curve.

It would be very instructive to construct similar global models for the higher order multi-spaces, as well as understanding their topological structure.

\section{The Calculus of Finite Differences.}

The proof of Theorem 2.3 requires the introduction of coordinate charts on $M^{(n)}$. Just as the local coordinates on $\mathrm{J}^{n}$ are provided by the coefficients of Taylor polynomials, the local coordinates on $M^{(n)}$ are provided by the coefficients of interpolating polynomials. These are most conveniently written in terms of the classical divided differences of numerical interpolation theory, $[\mathbf{9}, \mathbf{2 5}]$.

We work in a local coordinate chart $z=(x, u)$ on $M$. For ease of notation, we identify the coordinate chart with $M$ itself. An $(n+1)$-pointed graph consists of the graph of a smooth function $u=f(x)$ together with $(n+1)$ points $z_{i}=\left(x_{i}, f\left(x_{i}\right)\right)$ thereon. Again, it is worth emphasizing that we allow some or all of the mesh points $x_{0}, \ldots, x_{n} \in \mathbb{R}$ to coincide. The multi-jets of $(n+1)$-pointed graphs will form an open, dense submanifold $M_{\Gamma}^{(n)} \subset M^{(n)}$. The missing part $M^{(n)} \backslash M_{\Gamma}^{(n)}$ consists of multi-jets of $(n+1)$-pointed curves with either vertical tangents at repeated points, or having two or more distinct points lying on the same vertical line $\{x=c\}$.

We define the classical divided differences $\left[z_{0} z_{1} \ldots z_{k}\right]$ by the standard recursive rule

$$
\left[z_{0} z_{1} \ldots z_{k-1} z_{k}\right]=\frac{\left[z_{0} z_{1} z_{2} \ldots z_{k-2} z_{k}\right]-\left[z_{0} z_{1} z_{2} \ldots z_{k-2} z_{k-1}\right]}{x_{k}-x_{k-1}}, \quad\left[z_{j}\right]=u_{j} .
$$

The divided differences are well-defined provided no two points lie on the same vertical line. They are, in fact, symmetric functions of their arguments $z_{i}$. 
Remark: Classically, one employs the simpler notation $\left[u_{0} u_{1} \ldots u_{k}\right]$ for the divided difference $\left[z_{0} z_{1} \ldots z_{k}\right]$. However, the classical notation is ambiguous since it assumes that the mesh $x_{0}, \ldots, x_{n}$ is fixed throughout. Because we are regarding the independent and dependent variables on the same footing - and, indeed, are allowing changes of variables that scramble the two - it is important to adopt an unambiguous divided difference notation here.

The classical Newton interpolating polynomial constitutes the multi-space counterpart of the Taylor polynomial in jet space. Indeed, it reduces to the Taylor polynomial when all points are coincident.

Proposition 3.1. If $z_{0}, \ldots, z_{n}$ are $n+1$ distinct points, no two of which lie on the same vertical line, then there is a unique interpolating polynomial

$$
\begin{aligned}
p_{n}(x)=\left[z_{0}\right]+\left(x-x_{0}\right) & {\left[z_{0} z_{1}\right]+\left(x-x_{0}\right)\left(x-x_{1}\right)\left[z_{0} z_{1} z_{2}\right]+\cdots } \\
+ & \left(x-x_{0}\right)\left(x-x_{1}\right) \cdots\left(x-x_{n-1}\right)\left[z_{0} z_{1} \ldots z_{n}\right]
\end{aligned}
$$

of degree $\leq n$ that satisfies $u_{k}=p\left(x_{k}\right)$ for $k=0, \ldots, n$.

Divided differences are initially defined only for distinct points $z_{k}$. Requiring the points to lie on a smooth curve (graph) allows us to extend the definitions to cases when two or more points are coincident. To emphasize that the resulting "confluent divided differences" depend on the underlying curve (or function) we sometimes write $\left[z_{0} z_{1} \ldots z_{k}\right]_{C}$ instead of $\left[z_{0} z_{1} \ldots z_{k}\right]$.

Definition 3.2. Given an $(n+1)$-pointed graph $\mathbf{C}=\left(z_{0}, \ldots, z_{n} ; C\right)$, its divided differences are defined by $\left[z_{j}\right]_{C}=f\left(x_{j}\right)$, and

$$
\left[z_{0} z_{1} \ldots z_{k-1} z_{k}\right]_{C}=\lim _{z \rightarrow z_{k}} \frac{\left[z_{0} z_{1} z_{2} \ldots z_{k-2} z\right]_{C}-\left[z_{0} z_{1} z_{2} \ldots z_{k-2} z_{k-1}\right]_{C}}{x-x_{k-1}} .
$$

When taking the limit, the point $z=(x, f(x))$ must lie on the curve $C$, and take limiting values $x \rightarrow x_{k}$ and $f(x) \rightarrow f\left(x_{k}\right)$.

In the non-confluent case $z_{k} \neq z_{k-1}$ we can replace $z$ by $z_{k}$ directly in the difference quotient (3.3) and so ignore the limit. On the other hand, when all $k+1$ points coincide, the $k^{\text {th }}$ order confluent divided difference converges to

$$
\left[z_{0} \ldots z_{0}\right]=\frac{f^{(k)}\left(x_{0}\right)}{k !} .
$$

The generalization of the Newton interpolation formula (3.2) to the confluent case can now be stated.

Theorem 3.3. Let $x_{0}, \ldots, x_{n} \in \mathbb{R}$ be mesh points, and let $a_{0}, \ldots, a_{n} \in \mathbb{R}^{q}$. Define the $(n+1)$-pointed graph $\mathbf{C}=\left(z_{0}, \ldots, z_{n} ; C\right)$ where $C$ denotes the graph of the polynomial $p_{n}(x)=a_{0}+a_{1}\left(x-x_{0}\right)+a_{2}\left(x-x_{0}\right)\left(x-x_{1}\right)+\cdots+a_{n}\left(x-x_{0}\right)\left(x-x_{1}\right) \cdots\left(x-x_{n-1}\right)$, 
and $z_{k}=\left(x_{k}, p_{n}\left(x_{k}\right)\right) \in C$ for $k=0, \ldots, n$. Then the divided differences for $\mathbf{C}$ are equal to

$$
\left[z_{0} z_{1} \ldots z_{k}\right]_{C}=a_{k}, \quad k=0, \ldots, n .
$$

Theorem 3.4. Two $(n+1)$-pointed graphs $\mathbf{C}, \widetilde{\mathbf{C}}$ have $n^{\text {th }}$ order multi-contact if and only if they have the same divided differences:

$$
\left[z_{0} z_{1} \ldots z_{k}\right]_{C}=\left[z_{0} z_{1} \ldots z_{k}\right]_{\widetilde{C}}, \quad k=0, \ldots, n .
$$

In particular, $\widetilde{\mathbf{C}}=\left(z_{0}, \ldots, z_{n} ; \widetilde{C}\right)$ will have $n^{\text {th }}$ order multi-contact with the polynomial curve given by (3.5) if and only if $\widetilde{C}$ is the graph of a function of the form

$$
u=f(x)=p_{n}(x)+\left(x-x_{0}\right)\left(x-x_{1}\right) \cdots\left(x-x_{n}\right) h(x),
$$

where $h(x)$ is smooth.

We can now introduce local coordinates on the multi-graph subset $M_{\Gamma}^{(n)} \subset M^{(n)}$ of multi-space. They consist of the independent variables along with all the divided differences

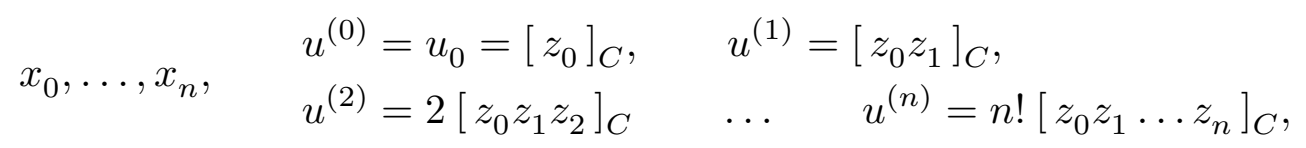

prescribed by $(n+1)$-pointed graphs $\mathbf{C}=\left(z_{0}, \ldots, z_{n} ; C\right)$. The $n$ ! factor is included so that $u^{(n)}$ agrees with the usual derivative coordinate when restricted to $\mathrm{J}^{n}$, cf. (3.4). For noncoincident points $\left(z_{0}, \ldots, z_{n}\right) \in M^{\diamond(n+1)}$, the divided difference coordinates are uniquely characterized by the Newton interpolation formulae

$$
\begin{aligned}
u_{0} & =u^{(0)} \\
u_{1} & =u^{(0)}+u^{(1)}\left(x_{1}-x_{0}\right), \\
u_{2} & =u^{(0)}+u^{(1)}\left(x_{2}-x_{0}\right)+\frac{u^{(2)}}{2}\left(x_{2}-x_{1}\right)\left(x_{2}-x_{0}\right), \\
& \vdots \\
u_{n}= & u^{(0)}+u^{(1)}\left(x_{2}-x_{0}\right)+\cdots+\frac{u^{(n)}}{n !}\left(x-x_{0}\right)\left(x-x_{1}\right) \cdots\left(x-x_{n-1}\right) .
\end{aligned}
$$

In particular,

$$
\begin{aligned}
& u^{(1)}=\left[z_{0} z_{1}\right]=\frac{u_{1}-u_{0}}{x_{1}-x_{0}}, \\
& u^{(2)}=2\left[z_{0} z_{1} z_{2}\right]=2 \frac{\left(x_{1}-x_{0}\right) u_{2}+\left(x_{0}-x_{2}\right) u_{1}+\left(x_{2}-x_{1}\right) u_{0}}{\left(x_{1}-x_{0}\right)\left(x_{2}-x_{0}\right)\left(x_{2}-x_{1}\right)} .
\end{aligned}
$$

These formulae allow us to rewrite functions $F\left(z_{0}, \ldots, z_{n}\right)$ on $M^{\diamond(n+1)}$ in terms of the multi-space divided difference coordinates, and thereby extend them to the jet and multijet subspaces.

Theorem 3.4 implies that the divided differences are uniquely determined by the multijet of a multi-pointed curve, and so provide local coordinates on $M^{(n)}$. The proof that 
the change of divided difference coordinates is smooth on the overlap of coordinate charts proceeds indirectly. One first proves that the divided differences depend smoothly on their arguments, even under smooth deformations of the underlying function as well as the points themselves. Note that, under deformations, coincident points may split apart, while distinct points may coalesce.

Lemma 3.5. Let $\mathbf{C}(t)=\left(z_{0}(t), \ldots, z_{n}(t) ; C(t)\right), t \in \mathbb{R}$, be a smoothly varying oneparameter family of $(n+1)$-pointed graphs. Then the corresponding divided differences $p(t)=\left[z_{0}(t), \ldots, z_{k}(t)\right]_{C(t)}$ are smooth functions of $t$.

One then invokes the following theorem due to Boman, [1], to complete the proof of smoothness of the changes of coordinates.

Theorem 3.6. If $f: W \rightarrow \mathbb{R}$ is a function defined on an open set $W \subset \mathbb{R}^{m}$ such that $f(z(t))$ is smooth for every smooth curve $z: \mathbb{R} \rightarrow W$, then $f(z)$ is a smooth function.

\section{Numerical Approximations.}

A smooth function $\Delta: \mathrm{J}^{n} \rightarrow \mathbb{R}$ on (an open subset of) the jet space, written $\Delta\left(x, u^{(n)}\right)$, is known as a differential function. These include individual derivatives, as well as more complicated combinations such as the Laplacian, the Euclidean curvature, general differential invariants, etc. Any system of differential equations (or, even more generally, a system of differential algebraic equations) is (locally) defined by the vanishing of one or more differential functions:

$$
\Delta_{1}\left(x, u^{(n)}\right)=\cdots=\Delta_{k}\left(x, u^{(n)}\right)=0 .
$$

To implement a numerical solution to the system (4.1) by finite difference methods, one relies on suitable discrete approximations to each of its defining differential functions $\Delta_{\nu}$, and this requires extending the differential functions from the jet space to the associated multi-space, in accordance with the following definition.

Definition 4.1. Let $M$ be a Riemannian manifold with metric $\|\cdot\|$. Let $N \subset M$ be a closed submanifold and $H: N \rightarrow \mathbb{R}$ a smooth function on $N$. We call $F: M \rightarrow \mathbb{R}$ an order $k$ extension of $H$ if for each compact $K \subset M$ there exists a constant $C>0$ so that

$$
|F(x)-H(y)| \leq C\|x-y\|^{k}, \quad x \in K,
$$

where $y \in N$ is the closest point on $N$ to $x$.

The definition is clearly independent of any particular choice of Riemannian metric on $M$. If we introduce local coordinates $z=(x, y)$ so that $N=\{x=0\}$, then (4.2) takes the form

$$
F(x, y)=H(y)+\mathrm{O}\left(\|x\|^{k}\right)
$$

and so can be checked via a straightforward Taylor expansion of $F$ on the submanifold.

Definition 4.2. An $(n+1)$-point numerical approximation of order $k$ to a differential function $\Delta: \mathrm{J}^{n} \rightarrow \mathbb{R}$ is a $k^{\text {th }}$ order extension $F: M^{(n)} \rightarrow \mathbb{R}$ of $\Delta$ to multi-space, based on the inclusion $\mathrm{J}^{n} \subset M^{(n)}$. 
In practice, one calculates the numerical approximation on the off-diagonal part $M^{\diamond(n+1)} \subset M^{(n)}$ where the points are non-coincident, and then relies on smoothness to ensure that it gives a reasonable approximation to the differential function as the points coalesce. In practice, $\Delta$ may only be defined on an open subset of $\mathrm{J}^{n}$, and the numerical approximation $F$ need only be defined in a neighborhood of the domain of definition of $\Delta$. Let us convince the reader that Definition 4.2 is a legitimate geometric reformulation of standard numerical approximation ideas.

The simplest illustration of Definition 4.2 is provided by the divided difference coordinates (3.8). Each divided difference $u^{(n)}$ forms an $(n+1)$-point numerical approximation to the $n^{\text {th }}$ order derivative coordinate on $\mathrm{J}^{n}$. The order of the approximation is $k=1$. More generally, any differential function $\Delta\left(x, u, u^{(1)}, \ldots u^{(n)}\right)$ can immediately be given an $(n+1)$-point numerical approximation $F=\Delta\left(x_{0}, u^{(0)}, u^{(1)}, \ldots u^{(n)}\right)$ by replacing each derivative by its divided difference coordinate approximation. However, these are by no means the only numerical approximations possible.

The order of such a numerical approximation is, in accordance with classical computations, determined by examination of its Taylor series. For example, expanding the first order divided difference about $x_{0}$, we find

$$
u^{(1)}=\left[z_{0} z_{1}\right]_{C}=\frac{f\left(x_{1}\right)-f\left(x_{0}\right)}{x_{1}-x_{0}}=f^{\prime}\left(x_{0}\right)+\frac{f^{\prime \prime}\left(x_{0}\right)}{2}\left(x_{1}-x_{0}\right)+\frac{f^{\prime \prime \prime}\left(x_{0}\right)}{6}\left(x_{1}-x_{0}\right)+\cdots .
$$

The degree of the error term, which in this case is 1 , is the order of approximation. To obtain higher order approximations, we need to include more data points, and the Taylor series provides a systematic method of construction. For example, on three-pointed graph, we have the first order divided difference approximation

$$
u^{(2)}=2\left[z_{0} z_{1} z_{2}\right]=2 \frac{\left(x_{1}-x_{0}\right)\left(u_{2}-u_{0}\right)-\left(x_{2}-x_{0}\right)\left(u_{1}-u_{0}\right)}{\left(x_{1}-x_{0}\right)\left(x_{2}-x_{0}\right)\left(x_{2}-x_{1}\right)}
$$

to the second derivative

$$
u^{(2)}=f^{\prime \prime}\left(x_{0}\right)+\frac{2}{3} f^{\prime \prime \prime}\left(x_{0}\right)\left(x_{1}+x_{2}-2 x_{0}\right)+\cdots .
$$

Replacing $f^{\prime \prime}\left(x_{0}\right)$ by $u^{(2)}$ in the expansion (4.3) leads to second order approximation

$$
\tilde{u}^{(1)}=u^{(1)}+\frac{1}{2}\left(x_{1}-x_{0}\right) u^{(2)}=\frac{\left(x_{1}-x_{0}\right)^{2}\left(u_{2}-u_{0}\right)-\left(x_{2}-x_{0}\right)^{2}\left(u_{1}-u_{0}\right)}{\left(x_{1}-x_{0}\right)\left(x_{2}-x_{0}\right)\left(x_{1}-x_{2}\right)}
$$

to the first derivative:

$$
\tilde{u}^{(1)}=f^{\prime}\left(x_{0}\right)-\frac{1}{6} f^{\prime \prime \prime}\left(x_{0}\right)\left(x_{1}-x_{0}\right)\left(x_{2}-x_{0}\right)+\cdots .
$$

When restricted to equally spaced mesh points $x_{1}=x_{0}-h, x_{2}=x_{0}+h$, we find

$$
\tilde{u}^{(1)}=\frac{u_{2}-u_{1}}{x_{2}-x_{1}}=\frac{f\left(x_{0}+h\right)-f\left(x_{0}-h\right)}{2 h}
$$

reduces to the familiar centered difference approximation to $f^{\prime}\left(x_{0}\right)$. Note that the approximation (4.4) is not symmetric under permutations of the points. 


\section{Multi-Invariants.}

We now introduce an $r$-dimensional Lie group $G$ which acts smoothly on $M$. Since $G$ evidently maps multi-pointed curves to multi-pointed curves while preserving the multicontact equivalence relation, it induces an action on the multi-space $M^{(n)}$ that will be called the $n^{\text {th }}$ multi-prolongation of $G$ and denoted by $G^{(n)}$. On the jet subset $\mathrm{J}^{n} \subset M^{(n)}$ the multi-prolonged action reduced to the usual jet space prolongation of our transformation group, [27]. On the other hand, on the off-diagonal part $M^{\diamond(n+1)} \subset M^{(n)}$ the action coincides with the $(n+1)$-fold Cartesian product action of $G$ on $M^{\times(n+1)},[\mathbf{2 9}]$.

Recall that a differential invariant is a function $I: \mathrm{J}^{n} \rightarrow \mathbb{R}$ which is invariant under the prolonged action of $G$ on the jet space $\mathrm{J}^{n}$. Similarly, a joint invariant is a function $J: M^{\times(n+1)} \rightarrow \mathbb{R}$ on the Cartesian product space which is invariant under the product action of $G$, cf. [29]. In this vein, we define a multi-invariant to be a function $K: M^{(n)} \rightarrow \mathbb{R}$ on multi-space which is invariant under the multi-prolonged action of $G^{(n)}$. The restriction of a multi-invariant $K$ to jet space will be a differential invariant, $I=K \mid \mathrm{J}^{n}$, while restriction to $M^{\diamond(n+1)}$ will define a joint invariant $J=K \mid M^{\diamond(n+1)}$. Smoothness of $K$ will imply that the joint invariant $J$ is an invariant $n^{\text {th }}$ order numerical approximation to the differential invariant $I$. Moreover, every invariant finite difference numerical approximation to the differential invariant $I$ arises in this manner. Thus, the theory of multi-invariants is the theory of invariant numerical approximations! The basic idea of replacing differential invariants by joint invariants forms the foundation of Dorodnitsyn's approach to invariant numerical algorithms, $[\mathbf{1 1}, \mathbf{1 2}]$, and also the invariant numerical approximations of differential invariant signatures in computer vision, $[\mathbf{2}, \mathbf{5}, \mathbf{6}, \mathbf{2 9}]$.

Furthermore, the restriction of a multi-invariant to an intermediate multi-jet subspace, as in (2.1), will define a joint differential invariant, $[\mathbf{2 9}]$ - also known as a semi-differential invariant in the computer vision literature, $[\mathbf{1 0}, \mathbf{2 6}]$. The approximation of differential invariants by joint differential invariants is, therefore, based on the extension of the differential invariant from the jet space to a suitable multi-jet subspace (2.1). The invariant numerical approximations to joint differential invariants are, in turn, obtained by extending them from the multi-jet subspace to the entire multi-space. Thus, multi-invariants also include invariant semi-differential approximations to differential invariants as well as joint invariant numerical approximations to differential invariants and semi-differential invariants - all in one seamless geometric framework.

Consequently, for symmetry-based numerical analysis, the construction of the requisite multi-invariants becomes a problem of paramount importance. The recently developed general theory of moving frames, $[\mathbf{1 5}, \mathbf{2 9}]$, provides a powerful, direct method for constructing invariants of very general transformation group actions. To apply this method here, we begin with a brief review of the basic moving frame constructions.

Definition 5.1. Given a finite-dimensional Lie group $G$ acting smoothly on a manifold $M$, a moving frame is a smooth, $G$-equivariant map $\rho: M \rightarrow G$.

The group $G$ acts on itself by left or right multiplication. Classical moving frames, $[\mathbf{7}, \mathbf{1 7}]$, which are all included in this general definition, rely on the left action, but, in practice, the right versions are often easier to compute, and will be the version of choice 
here. Right-equivariance requires

$$
\rho(g \cdot z)=\rho(z) \cdot g^{-1} \quad \text { for all } \quad z \in M, \quad g \in G .
$$

The classical left-equivariant moving frame $\widetilde{\rho}(z)=\rho(z)^{-1}$ may be simply obtained by applying the group inversion.

Theorem 5.2. A moving frame exists in a neighborhood of a point $z \in M$ if and only if $G$ acts freely and regularly near $z$.

Freeness requires that every point in $M$ has trivial isotropy subgroup, $G_{z}=\{e\}$. Therefore, the group orbits are all of dimension $r=\operatorname{dim} G$. Regularity requires that the orbits form a regular foliation; see $[\mathbf{1 5}, \mathbf{2 7}]$ for details.

The practical implementation of the moving frame construction is based on Cartan's method of normalization, $[\mathbf{7}, \mathbf{1 5}]$, which relies on the choice of a cross-section to the $r$ dimensional group orbits.

Theorem 5.3. If $G$ acts freely, regularly on $M$, and $\mathcal{K} \subset M$ is a cross-section to the group orbits, then the map $\rho: M \rightarrow G$ that sends $z \in M$ to the unique group element $g=\rho(z)$ that maps $z$ to the cross-section, $g \cdot z=\rho(z) \cdot z \in \mathcal{K}$, defines a right moving frame.

One usually chooses a local coordinate cross-section $\mathcal{K}=\left\{z_{1}=c_{1}, \ldots, z_{r}=c_{r}\right\}$, where the first $r$, say, of the coordinates $z=\left(z_{1}, \ldots, z_{m}\right)$ on $M$ are set equal to suitably chosen constants. If we write out the local coordinate formulae $w(g, z)=g \cdot z$ for the group transformations, then the corresponding right moving frame $g=\rho(z)$ is obtained by solving the normalization equations

$$
w_{1}(g, z)=c_{1}, \quad \ldots \quad w_{r}(g, z)=c_{r},
$$

for the group parameters $g=\left(g_{1}, \ldots, g_{r}\right)$ in terms of the coordinates $z=\left(z_{1}, \ldots, z_{m}\right)$. When we substitute the moving frame expressions $g=\rho(z)$ into the transformation formulae, the resulting functions $I_{\nu}(z)=w_{\nu}(\rho(z), z)$ are easily seen to be $G$-invariant. The first $r$ coincide with the normalization constants, $I_{1}(z)=c_{1}, \ldots, I_{r}(z)=c_{r}$, while the remaining $m-r$ provide a system of fundamental invariants for the group action.

Theorem 5.4. If $g=\rho(z)$ is the moving frame solution to the normalization equations (5.1), then $I_{r+1}(z)=w_{r+1}(\rho(z), z), \ldots, I_{m}(z)=w_{m}(\rho(z), z)$ form a complete system of functionally independent invariants.

The moving frame construction provides an added bonus - a canonical way to associate an invariant with any function.

Definition 5.5. The invariantization of a scalar function $F: M \rightarrow \mathbb{R}$ with respect to a right moving frame $\rho$ is the the invariant function $I=\iota(F)$ defined by $I(z)=F(\rho(z) \cdot z)$.

In particular, if $I(z)$ is an invariant, then $\iota(I)=I$. Therefore, invariantization defines a canonical projection, depending on the moving frame, from functions to invariants. Geometrically, invariantization amounts to restricting the function to the cross-section and then requiring that the induced invariant be constant along the group orbits. 
So far, the moving frame construction has rested upon the hypothesis that the group action on $M$ is both regular and free. Most interesting group actions are not free. There are two common methods for making an (effective) group action free. In classical applications in geometry, $[\mathbf{7}, \mathbf{1 7}, \mathbf{2 2}]$, this is accomplished by prolonging the action to a jet space $\mathrm{J}^{n}$ of suitably high order; the consequential invariants are the classical differential invariants for the group, $[\mathbf{1 5}, \mathbf{2 7}]$. Alternatively, one may consider the product action of $G$ on a sufficiently large Cartesian product $M^{\times(n+1)}$; here, the invariants are joint invariants, [29], of particular interest in classical algebra, $[\mathbf{2 8}, \mathbf{3 3}]$. In neither case is there a general theorem guaranteeing the freeness and regularity of the prolonged or product actions, (indeed, there are counterexamples in the product case), but such pathologies never occur in practical examples. In our approach to invariant numerical approximations, we amalgamate the two methods by prolonging to an appropriate multi-space. The moving frame on the multi-space, which we refer to as a multi-frame, will lead us immediately to the required multi-invariants and hence a general, systematic construction for invariant numerical approximations to differential invariants. Any multi-frame $\rho^{(n)}: M^{(n)} \rightarrow G$ will evidently restrict to a classical moving frame $\rho^{(n)}: \mathrm{J}^{n} \rightarrow G$ on the jet space along with a compatible product frame $\rho^{\diamond(n+1)}: M^{\diamond(n+1)} \rightarrow G$.

In local coordinates, we use $w_{k}=\left(y_{k}, v_{k}\right)=g \cdot z_{k}$ to denote the transformation formulae for the individual points on a multi-pointed curve. The multi-prolonged action on the divided difference coordinates gives

$$
\begin{aligned}
y_{0}, \ldots, y_{n}, & v^{(0)}=v_{0}=\left[w_{0}\right], & v^{(1)}=\left[w_{0} w_{1}\right], \\
v^{(2)}=2\left[w_{0} w_{1} w_{2}\right], & \ldots & v^{(n)}=n !\left[w_{0}, \ldots, w_{n}\right],
\end{aligned}
$$

where the formulae are most easily computed via the difference quotients

$$
\left[w_{0} w_{1} \ldots w_{k-1} w_{k}\right]=\frac{\left[w_{0} w_{1} w_{2} \ldots w_{k-2} w_{k}\right]-\left[w_{0} w_{1} w_{2} \ldots w_{k-2} w_{k-1}\right]}{y_{k}-y_{k-1}}, \quad\left[w_{j}\right]=v_{j},
$$

and then taking appropriate limits to cover the case of coalescing points. Inspired by the constructions in [15], we will refer to (5.2) as the lifted divided difference invariants.

To compute a multi-frame, we need to normalize by choosing a cross-section to the group orbits in $M^{(n)}$, which amounts to setting $r=\operatorname{dim} G$ of the lifted divided difference invariants (5.2) equal to suitably chosen constants. An important observation is that in order to obtain the limiting differential invariants, we must require our local cross-section to pass through the jet space, and define, by intersection, a cross-section for the prolonged action on $\mathrm{J}^{n}$. This compatibility constraint implies that we are only allowed to normalize the first lifted independent variable $y_{0}=c_{0}$. If we try to normalize $y_{1}$ then we must either set $y_{1}=c_{0}=y_{0}$, and the cross-section would only be valid for coincident points $w_{1}=w_{0}$ which would prevent us from extending it to the non-coincident case required for constructing invariant numerical approximations, or set $y_{1}=c_{1} \neq c_{0}$, and this would prevent the points $w_{0}$ and $w_{1}$ from coalescing, so our moving frame could not be restricted to the jet subspace!

With the aid of the multi-frame, the most direct construction of the requisite multiinvariants and associated invariant numerical differentiation formulae is through the in- 
variantization of the original finite difference quotients (3.1). Substituting the multi-frame formulae for the group parameters into the lifted coordinates (5.2) provides a complete system of multi-invariants on $M^{(n)}$; this follows immediately from Theorem 5.4. We denote the fundamental multi-invariants by

$$
y_{i} \longmapsto H_{i}=\iota\left(x_{i}\right), \quad v^{(n)} \longmapsto K^{(n)}=\iota\left(u^{(n)}\right),
$$

where $\iota$ denotes the invariantization map associated with the multi-frame. The fundamental differential invariants for the prolonged action of $G$ on $\mathrm{J}^{n}$ can all be obtained by restriction, so that $I^{(n)}=K^{(n)} \mid \mathrm{J}^{n}$. On the jet space, the points are coincident, and so the multi-invariants $H_{i}$ will all restrict to the same differential invariant $c_{0}=H=H_{i} \mid \mathrm{J}^{n}$ - the normalization value of $y_{0}$. On the other hand, the fundamental joint invariants on $M^{\diamond(n+1)}$ are obtained by restricting the multi-invariants $H_{i}=\iota\left(x_{i}\right)$ and $K_{i}=\iota\left(u_{i}\right)$. The multi-invariants can computed by using a multi-invariant divided difference recursion

$$
\left[I_{j}\right]=K_{j}=\iota\left(u_{j}\right) \quad\left[I_{0} \ldots I_{k}\right]=\iota\left(\left[z_{0} z_{1} \ldots z_{k}\right]\right)=\frac{\left[I_{0} \ldots I_{k-2} I_{k}\right]-\left[I_{0} \ldots I_{k-2} I_{k-1}\right]}{H_{k}-H_{k-1}},
$$

and then relying on continuity to extend the formulae to coincident points. The multiinvariants

$$
K^{(n)}=n !\left[I_{0} \ldots I_{n}\right]=\iota\left(u^{(n)}\right)
$$

define the fundamental first order invariant numerical approximations to the differential invariants $I^{(n)}$. Higher order invariant numerical approximations can be obtained by invariantization of the higher order divided difference approximations, cf. (4.4). The moving frame construction has a significant advantage over the infinitesimal approach used by Dorodnitsyn, $[\mathbf{1 1}, \mathbf{1 2}]$, in that it does not require the solution of partial differential equations in order to construct the multi-invariants.

Given a $G$-invariant differential equation

$$
\Delta\left(x, u^{(n)}\right)=0,
$$

we can invariantize the left hand side to rewrite the differential equation in terms of the fundamental differential invariants:

$$
\iota\left(\Delta\left(x, u^{(n)}\right)\right)=\Delta\left(H, I^{(0)}, \ldots, I^{(n)}\right)=0 .
$$

The invariant finite difference approximation to the differential equation is then obtained by replacing the differential invariants $I^{(k)}$ by their multi-invariant counterparts $K^{(k)}$ :

$$
\Delta\left(c_{0}, K^{(0)}, \ldots, K^{(n)}\right)=0 .
$$

Lack of space precludes a more detailed development and analysis of these ideas here, and we close with two simple illustrative examples.

Example 5.6. Consider the elementary action

$$
(x, u) \longmapsto\left(\lambda^{-1} x+a, \lambda u+b\right)
$$


of the three-parameter similarity group $G=\mathbb{R}^{2} \ltimes \mathbb{R}$ on $M=\mathbb{R}^{2}$. To obtain the multiprolonged action, we compute the divided differences (5.2) of the basic lifted invariants

$$
y_{k}=\lambda^{-1} x_{k}+a, \quad v_{k}=\lambda u_{k}+b .
$$

We find

$$
v^{(1)}=\left[w_{0} w_{1}\right]=\frac{v_{1}-v_{0}}{y_{1}-y_{0}}=\lambda^{2} \frac{u_{1}-u_{0}}{x_{1}-x_{0}}=\lambda^{2}\left[z_{0} z_{1}\right]=\lambda^{2} u^{(1)} .
$$

More generally,

$$
v^{(n)}=\lambda^{n+1} u^{(n)}, \quad n \geq 1 .
$$

Note that we may compute the multi-space transformation formulae assuming initially that the points are distinct, and then extending to coincident cases by continuity. (In fact, this gives an alternative method for computing the standard jet space prolongations of group actions!) In particular, when all the points coincide, each $u^{(n)}$ reduces to the $n^{\text {th }}$ order derivative coordinate, and (5.9) reduces to the prolonged action of $G$ on $\mathrm{J}^{n}$. We choose the normalization cross-section defined by

$$
y_{0}=0, \quad v_{0}=0, \quad v^{(1)}=1,
$$

which, upon solving for the group parameters, leads to the basic moving frame

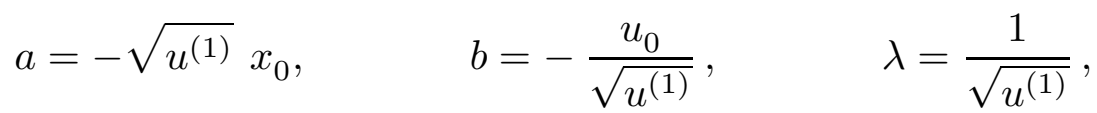

where, for simplicity, we restrict to the subset where $u^{(1)}=\left[z_{0} z_{1}\right]>0$. The fundamental joint similarity invariants are obtained by substituting these formulae into

$$
\begin{aligned}
& y_{k} \longmapsto H_{k}=\left(x_{k}-x_{0}\right) \sqrt{u^{(1)}}=\left(x_{k}-x_{0}\right) \sqrt{\frac{u_{1}-u_{0}}{x_{1}-x_{0}}}, \\
& v_{k} \longmapsto K_{k}=\frac{u_{k}-u_{0}}{\sqrt{u^{(1)}}}=\left(u_{k}-u_{0}\right) \sqrt{\frac{x_{1}-x_{0}}{u_{1}-u_{0}}},
\end{aligned}
$$

both of which reduce to the trivial zero differential invariant on $\mathrm{J}^{n}$. Higher order multiinvariants are obtained by substituting (5.10) into the lifted invariants (5.9), leading to

$$
K^{(n)}=\frac{u^{(n)}}{\left(u^{(1)}\right)^{(n+1) / 2}}=\frac{n !\left[z_{0} z_{1} \ldots z_{n}\right]}{\left[z_{0} z_{1} z_{2}\right]^{(n+1) / 2}} .
$$

In the limit, these reduce to the differential invariants $I^{(n)}=\left(u^{(1)}\right)^{-(n+1) / 2} u^{(n)}$, and so $K^{(n)}$ give the desired similarity-invariant, first order numerical approximations. To construct an invariant numerical scheme for any similarity-invariant ordinary differential equation

$$
\Delta\left(x, u, u^{(1)}, u^{(2)}, \ldots u^{(n)}\right)=0
$$

we merely invariantize the defining differential function, leading to the general similarityinvariant numerical approximation

$$
\Delta\left(0,0,1, K^{(2)}, \ldots, K^{(n)}\right)=0 .
$$


Example 5.7. The action of the proper Euclidean group of $\mathrm{SE}(2)$ on $M=\mathbb{R}^{2}$ given by

$$
(y, v)=g \cdot(x, u)=(x \cos \theta-u \sin \theta+a, x \sin \theta+u \cos \theta+b)
$$

forms the foundation of the Euclidean geometry of planar curves. The multi-prolonged action is free on $M^{(n)}$ for $n \geq 1$, and we can thereby determine a first order multi-frame and use it to completely classify Euclidean multi-invariants. The first order transformation formulae are

$$
\begin{array}{ll}
y_{0}=x_{0} \cos \theta-u_{0} \sin \theta+a, & v_{0}=x_{0} \sin \theta+u_{0} \cos \theta+b, \\
y_{1}=x_{1} \cos \theta-u_{1} \sin \theta+a, & v^{(1)}=\frac{\sin \theta+u^{(1)} \cos \theta}{\cos \theta-u^{(1)} \sin \theta},
\end{array}
$$

where $u^{(1)}=\left[z_{0} z_{1}\right]$. Normalization based on the cross-section $y_{0}=v_{0}=v^{(1)}=0$ results in the right moving frame

$$
\begin{aligned}
& a=-x_{0} \cos \theta+u_{0} \sin \theta=-\frac{x_{0}+u^{(1)} u_{0}}{\sqrt{1+\left(u^{(1)}\right)^{2}}}, \\
& b=-x_{0} \sin \theta-u_{0} \cos \theta=\frac{x_{0} u^{(1)}-u_{0}}{\sqrt{1+\left(u^{(1)}\right)^{2}}},
\end{aligned}
$$

(Actually, the angular coordinate $\theta$ is not quite uniquely specified by this cross-section, since $\theta+\pi$ also solves the normalization equations. This ambiguity can be resolved either by restricting to oriented curves, or by prolonging to $M^{(2)}$. If we wish to also include reflections, then there is a second sign ambiguity that must be taken into account - see [29] for full details.) Substituting the moving frame formulae (5.13) into the lifted divided differences results in a complete system of (oriented) Euclidean multi-invariants. These are easily computed by beginning with the fundamental joint invariants $I_{k}=\left(H_{k}, K_{k}\right)=$ $\iota\left(x_{k}, u_{k}\right)$, where

$$
\begin{aligned}
& y_{k} \longmapsto H_{k}=\frac{\left(x_{k}-x_{0}\right)+u^{(1)}\left(u_{k}-u_{0}\right)}{\sqrt{1+\left(u^{(1)}\right)^{2}}}=\left(x_{k}-x_{0}\right) \frac{1+\left[z_{0} z_{1}\right]\left[z_{0} z_{k}\right]}{\sqrt{1+\left[z_{0} z_{1}\right]^{2}}}, \\
& v_{k} \longmapsto K_{k}=\frac{\left(u_{k}-u_{0}\right)-u^{(1)}\left(x_{k}-x_{0}\right)}{\sqrt{1+\left(u^{(1)}\right)^{2}}}=\left(x_{k}-x_{0}\right) \frac{\left[z_{0} z_{k}\right]-\left[z_{0} z_{1}\right]}{\sqrt{1+\left[z_{0} z_{1}\right]^{2}}} .
\end{aligned}
$$

The multi-invariants are obtained by forming divided difference quotients

$$
\left[I_{0} I_{k}\right]=\frac{K_{k}-K_{0}}{H_{k}-H_{0}}=\frac{K_{k}}{H_{k}}=\frac{\left(x_{k}-x_{1}\right)\left[z_{0} z_{1} z_{k}\right]}{1+\left[z_{0} z_{k}\right]\left[z_{0} z_{1}\right]},
$$

where, in particular, $I^{(1)}=\left[I_{0} I_{1}\right]=0$. The second order multi-invariant

$$
\begin{aligned}
I^{(2)} & =2\left[I_{0} I_{1} I_{2}\right]=2 \frac{\left[I_{0} I_{2}\right]-\left[I_{0} I_{1}\right]}{H_{2}-H_{1}}=\frac{2\left[z_{0} z_{1} z_{2}\right] \sqrt{1+\left[z_{0} z_{1}\right]^{2}}}{\left(1+\left[z_{0} z_{1}\right]\left[z_{1} z_{2}\right]\right)\left(1+\left[z_{0} z_{1}\right]\left[z_{0} z_{2}\right]\right)} \\
& =\frac{u^{(2)} \sqrt{1+\left(u^{(1)}\right)^{2}}}{\left[1+\left(u^{(1)}\right)^{2}+\frac{1}{2} u^{(1)} u^{(2)}\left(x_{2}-x_{0}\right)\right]\left[1+\left(u^{(1)}\right)^{2}+\frac{1}{2} u^{(1)} u^{(2)}\left(x_{2}-x_{1}\right)\right]}
\end{aligned}
$$


provides a Euclidean-invariant numerical approximation to the Euclidean curvature:

$$
\lim _{z_{1}, z_{2} \rightarrow z_{0}} I^{(2)}=\kappa=\frac{u^{(2)}}{\left(1+\left(u^{(1)}\right)^{2}\right)^{3 / 2}} .
$$

Similarly, the third order multi-invariant

$$
I^{(3)}=6\left[I_{0} I_{1} I_{2} I_{3}\right]=6 \frac{\left[I_{0} I_{1} I_{3}\right]-\left[I_{0} I_{1} I_{2}\right]}{H_{3}-H_{2}}
$$

will form a Euclidean-invariant approximation for the normalized differential invariant $\kappa_{s}=\iota\left(u_{x x x}\right)$, the derivative of curvature with respect to arc length, $[\mathbf{5}, \mathbf{1 5}]$.

To compare these with the invariant numerical approximations proposed in $[\mathbf{6}, \mathbf{5}, \mathbf{2}]$, we reformulate the divided difference formulae in terms of the geometrical configurations of the four distinct points $z_{0}, z_{1}, z_{2}, z_{3}$ on our curve. We find

$$
\begin{aligned}
& H_{k}=\frac{\left(z_{1}-z_{0}\right) \cdot\left(z_{k}-z_{0}\right)}{\left\|z_{1}-z_{0}\right\|}=r_{k} \cos \phi_{k}, \\
& K_{k}=\frac{\left(z_{1}-z_{0}\right) \wedge\left(z_{k}-z_{0}\right)}{\left\|z_{1}-z_{0}\right\|}=r_{k} \sin \phi_{k},
\end{aligned} \quad\left[I_{0} I_{k}\right]=\tan \phi_{k}
$$

where

$$
r_{k}=\left\|z_{k}-z_{0}\right\|, \quad \quad \phi_{k}=\Varangle\left(z_{k}-z_{0}, z_{1}-z_{0}\right),
$$

denotes the distance and the angle between the indicated vectors. Therefore,

$$
\begin{aligned}
I^{(2)} & =2 \frac{\tan \phi_{2}}{r_{2} \cos \phi_{2}-r_{1}}, \\
I^{(3)} & =6 \frac{\left(r_{2} \cos \phi_{2}-r_{1}\right) \tan \phi_{3}-\left(r_{3} \cos \phi_{3}-r_{1}\right) \tan \phi_{2}}{\left(r_{2} \cos \phi_{2}-r_{1}\right)\left(r_{3} \cos \phi_{3}-r_{1}\right)\left(r_{3} \cos \phi_{3}-r_{2} \cos \phi_{2}\right)} .
\end{aligned}
$$

Interestingly, $I^{(2)}$ is not the same Euclidean approximation to the curvature that was used in $[\mathbf{5}, \mathbf{6}]$. The latter was based on the Heron formula for the radius of a circle through three points:

$$
I^{\star}=\frac{4 \Delta}{a b c}=\frac{2 \sin \phi_{2}}{\left\|z_{1}-z_{2}\right\|} .
$$

Here $\Delta$ denotes the area of the triangle connecting $z_{0}, z_{1}, z_{2}$ and

$$
a=r_{1}=\left\|z_{1}-z_{0}\right\|, \quad b=r_{2}=\left\|z_{2}-z_{0}\right\|, \quad c=\left\|z_{2}-z_{1}\right\|,
$$

are its side lengths. The ratio tends to a limit $I^{\star} / I^{(2)} \rightarrow 1$ as the points coalesce. The geometrical approximation (5.15) has the advantage that it is symmetric under permutations of the points; one can achieve the same thing by symmetrizing the divided difference version $I^{(2)}$. Furthermore, $I^{(3)}$ is an invariant approximation for the differential invariant $\kappa_{s}$, that, like the approximations constructed by Boutin, [2], converges properly for arbitrary spacings of the points on the curve.

Both $I^{(2)}$ and $I^{(3)}$ are first order numerical approximations to their respective differential invariants. One can form invariant approximations of higher order by invariantization 
of the higher order divided difference approximations to ordinary derivatives. For example, invariantization of the second order approximation

$$
\frac{\left\{\left(x_{2}-x_{0}\right)^{2}-\left(x_{1}-x_{0}\right)^{2}\right\}\left[z_{0} z_{3}\right]+\left\{\left(x_{1}-x_{0}\right)^{2}-\left(x_{3}-x_{0}\right)^{2}\right\}\left[z_{0} z_{2}\right]}{+\left\{\left(x_{3}-x_{0}\right)^{2}-\left(x_{2}-x_{0}\right)^{2}\right\}\left[z_{0} z_{1}\right]}
$$

to $f^{\prime \prime}\left(x_{0}\right)$ leads to a second order invariant numerical approximation to the Euclidean curvature:

$$
\frac{\left(r_{2}^{2} \cos ^{2} \phi_{2}-r_{1}^{2}\right) \tan \phi_{3}-\left(r_{3}^{2} \cos ^{2} \phi_{2}-r_{1}^{2}\right) \tan \phi_{2}}{\left(r_{2} \cos \phi_{2}-r_{1}\right)\left(r_{3} \cos \phi_{3}-r_{1}\right)\left(r_{3} \cos \phi_{3}-r_{2} \cos \phi_{2}\right)} .
$$

Lack of space precludes us from tackling any more substantial examples here. The interested reader may find the analysis of the planar equi-affine group $w=A z+b$, where $\operatorname{det} A=1$, a good challenge. This group action underlies the (equi-)affine geometry of planar curves, $[\mathbf{1 7}]$, and has been extensively used in computer vision; see $[\mathbf{5}, \mathbf{6}]$ and the references therein.

Acknowledgments: I would like to thank Peter Hydon for helpful comments on an earlier draft of this paper.

\section{References}

[1] Boman, J., Differentiability of a function and of its compositions with functions of one variable, Math. Scand. 20 (1967), 249-268.

[2] Boutin, M., Numerically invariant signature curves, Int. J. Computer Vision 40 (2000), 235-248.

[3] Budd, C.J., and Collins, C.B., Symmetry based numerical methods for partial differential equations, in: Numerical analysis 1997, D.F. Griffiths, D.J. Higham and G.A. Watson, eds., Pitman Res. Notes Math., vol. 380, Longman, Harlow, 1998, pp. 16-36.

[4] Budd, C.J., and Iserles, A., Geometric integration: numerical solution of differential equations on manifolds, Phil. Trans. Roy. Soc. London A 357 (1999), 945-956.

[5] Calabi, E., Olver, P.J., Shakiban, C., Tannenbaum, A., and Haker, S., Differential and numerically invariant signature curves applied to object recognition, Int. J. Computer Vision 26 (1998), 107-135.

[6] Calabi, E., Olver, P.J., and Tannenbaum, A., Affine geometry, curve flows, and invariant numerical approximations, Adv. in Math. 124 (1996), 154-196.

[7] Cartan, É., La Méthode du Repère Mobile, la Théorie des Groupes Continus, et les Espaces Généralisés, Exposés de Géométrie No. 5, Hermann, Paris, 1935.

[8] Channell, P.J., and Scovel, C., Symplectic integration of Hamiltonian systems, Nonlinearity 3 (1990), 231-259.

[9] Davis, P.J., Interpolation and Approximation, Dover Publ. Inc., New York, 1975.

[10] Dhooghe, P.F., Multilocal invariants, in: Geometry and Topology of Submanifolds, VIII, F. Dillen, B. Komrakov, U. Simon, I. Van de Woestyne, and L.

Verstraelen, eds., World Sci. Publishing, Singapore, 1996, pp. 121-137. 
[11] Dorodnitsyn, V.A., Transformation groups in net spaces, J. Sov. Math. 55 (1991), 1490-1517.

[12] Dorodnitsyn, V.A., Finite difference models entirely inheriting continuous symmetry of original differential equations, Int. J. Mod. Phys. C 5 (1994), 723-734.

[13] Ehresmann, C., Introduction à la théorie des structures infinitésimales et des pseudo-groupes de Lie, in: Géometrie Différentielle, Colloq. Inter. du Centre Nat. de la Rech. Sci., Strasbourg, 1953, pp. 97-110.

[14] Fels, M., and Olver, P.J., Moving coframes. I. A practical algorithm, Acta Appl. Math. 51 (1998), 161-213.

[15] Fels, M., and Olver, P.J., Moving coframes. II. Regularization and theoretical foundations, Acta Appl. Math. 55 (1999), 127-208.

[16] Griffiths, P.A., and Harris, J., Principles of Algebraic Geometry, John Wiley \& Sons, New York, 1978.

[17] Guggenheimer, H.W., Differential Geometry, McGraw-Hill, New York, 1963.

[18] Hironaka, H., Resolution of singularities of an algebraic variety over a field of characteristic zero, Ann. Math. 78 (1964), 109-326.

[19] Iarrobino, A., Hilbert scheme of points: overview of last ten years, Proc. Sympos. Pure Math. 46 (1987), 297-320.

[20] Iserles, A., Munthe-Kaas, H.Z., Nørsett, S.P., and Zanna, A., Lie group methods, Acta Numerica (2000), 215-365.

[21] Jaegers, P.J., Lie group invariant finite difference schemes for the neutron diffusion equation, Ph.D. Thesis, Los Alamos National Lab Report, LA-12791-T, 1994.

[22] Jensen, G.R., Higher Order Contact of Submanifolds of Homogeneous Spaces, Lecture Notes in Math., vol. 610, Springer-Verlag, New York, 1977.

[23] Lewis, D., and Simo, J.C., Conserving algorithms for the dynamics of Hamiltonian systems on Lie groups, J. Nonlin. Sci. 4 (1994), 253-299.

[24] McLachlan, R.I., Quispel, G.R.W., and Robidoux, N., Geometric integration using discrete gradients, Phil. Trans. Roy. Soc. London A 357 (1999), 1021-1045.

[25] Milne-Thompson, L.M., The Calculus of Finite Differences, Macmilland and Co., Ltd., London, 1951.

[26] Moons, T., Pauwels, E., Van Gool, L., and Oosterlinck, A., Foundations of semi-differential invariants, Int. J. Comput. Vision 14 (1995), 25-48.

[27] Olver, P.J., Applications of Lie Groups to Differential Equations, Second Edition, Graduate Texts in Mathematics, vol. 107, Springer-Verlag, New York, 1993.

[28] Olver, P.J., Classical Invariant Theory, London Math. Soc. Student Texts, vol. 44, Cambridge University Press, Cambridge, 1999.

[29] Olver, P.J., Joint invariant signatures, Found. Comput. Math. 1 (2001), 3-67.

[30] Olver, P.J., On multivariate interpolation, Stud. Appl. Math. 116 (2006), 201-240.

[31] Shokin, Y.I., The Method of Differential Approximation, Springer-Verlag, New York, 1983.

[32] van Beckum, F.P.H., and van Groesen, E., Discretizations conserving energy and other constants of the motion, in: Proc. ICIAM 87, Paris, 1987, pp. 17-35 .

[33] Weyl, H., Classical Groups, Princeton Univ. Press, Princeton, N.J., 1946. 